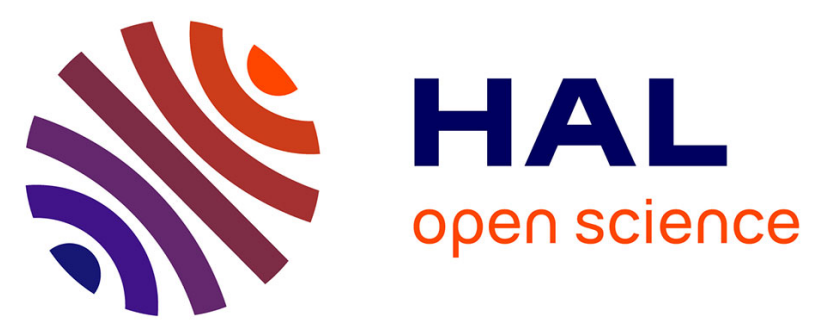

\title{
Experimental verification of neutron phenomenology in lead and transmutation by adiabatic resonance crossing in accelerator driven systems
}

\author{
H. Arnould, C.A. Bompas, R. del Moral, V. Lacoste, V. Vlachoudis, J. \\ Aleixandre, J. Bueno, E. Cerro, O. Gonzalez, J. Tamarit, et al.
}

\section{To cite this version:}

H. Arnould, C.A. Bompas, R. del Moral, V. Lacoste, V. Vlachoudis, et al.. Experimental verification of neutron phenomenology in lead and transmutation by adiabatic resonance crossing in accelerator driven systems. Physics Letters B, 1999, 458, pp.167-180. 10.1016/S0370-2693(99)00584-5 . in2p300002664

\section{HAL Id: in2p3-00002664 https://hal.in2p3.fr/in2p3-00002664}

Submitted on 26 Jul 1999

HAL is a multi-disciplinary open access archive for the deposit and dissemination of scientific research documents, whether they are published or not. The documents may come from teaching and research institutions in France or abroad, or from public or private research centers.
L'archive ouverte pluridisciplinaire HAL, est destinée au dépôt et à la diffusion de documents scientifiques de niveau recherche, publiés ou non, émanant des établissements d'enseignement et de recherche français ou étrangers, des laboratoires publics ou privés. 


\title{
EUROPEAN ORGANIZATION FOR NUCLEAR RESEARCH
}

CERN-LHC/98-12 (EET)

May 06,1999

\section{"Experimental Verification of Neutron Phenomenology in Lead and Transmutation by Adiabatic Resonance Crossing in Accelerator Driven Systems"}

\author{
A. Abánadesn,(§), J. Aleixandreb, S. Andriamonjec,a, A. Angelopoulosi, \\ A. Apostolakisj, H. Arnoulda, E. Belleg, C.A. Bompasa, D. Brozzic, J. Buenob, \\ S. Buonoc,h,(¥), F. Carminatic, F. Casagrande, ${ }^{c, e},(\mathbb{I})$, P. Cenninic, J.I. Collarc, (æ), \\ E. Cerro b, R. Del Morala, S. Díezm,(§), L. Dumps c, C. Eleftheriadisl, M. Embidi,d, \\ R. Fernándezc,d, J. Gálvezi, J. Garcían,d, C. Gelèsc, A. Giornig, E. Gonzálezd, \\ O. Gonzálezb, I. Goulasc, D. Heuerg, M. Hussonnoisf, Y. Kadic, P. Karaiskosi, \\ G. Kitis', R. Klapischc,(fi), P. Kokkask, V. Lacostea, C. Le Naourf, C. Lópezi, \\ J.M. Loiseauxg, J.M. Martínez-Valn, O. Méplang, H. Nifeneckerg, J. Oropesac, \\ I. Papadopoulos, P. Pavlopoulosk, E. Pérez-Encisoi, A. Pérez-Navarrom,(§), \\ M. Perladon, A. Placcic, M. Pozai, J.-P. Revolc, C. Rubbiac, J.A. Rubioc, L. Sakelliouj, \\ F. Saldañac, E. Savvidisl, F. Schusslerg, C. Sirvent $t^{i}$ J. Tamarit ${ }^{\text {, }}$, D. Trubert ${ }^{f}$, \\ A. Tzimal, J. B. Vianog, S. Vieira, V. Vlachoudis ${ }^{\mathrm{a}, \mathrm{l}}$, K. Zioutas!
}

(a) CEN, Bordeaux-Gradignan, France; (b) CEDEX, Madrid, Spain; (c) CERN, Geneva, Switzerland; (d) CIEMAT, Madrid, Spain; (e) INFN, Laboratori Nazionali di Frascati, Italy; (f) IPN, Orsay, France; (g) ISN, Grenoble, France; (h) Sincrotrone Trieste, Trieste, Italy; (i) Universidad Autonoma de Madrid, Madrid, Spain; (j) University of Athens, Athens, Greece; ( $k$ ) University of Basel, Basel Switzerland; (l) University of Thessaloniki, Thessaloniki, Greece; (m) Universidad Alfonso X el Sabio, Madrid, Spain; (n) Universidad Politécnica de Madrid, Madrid, Spain.

\begin{abstract}
Energy and space distributions of spallation neutrons (from 2.5 and $3.57 \mathrm{GeV} / \mathrm{c} C E R N$ proton beams) slowing down in a $3.3 \times 3.3 \times 3 \mathrm{~m}^{3}$ lead volume and neutron capture rates on long-lived fission fragments ${ }^{99} \mathrm{Tc}$ and ${ }^{129}$ I demonstrate that Adiabatic Resonance Crossing (ARC) can be used to eliminate efficiently such nuclear waste and validate innovative simulation.
\end{abstract}

(submitted to Phys. Lett. B)
(§) Present address LAESA, Zaragoza, Spain
$(¥)$ Present address CRS4, Cagliari, Italy
(I) Present address MIT, Cambridge, USA
(æ) Present address Université Paris VI, Paris, France
(fi) Also at CSNSM, IN2P3, Orsay, France 


\section{Introduction}

An Accelerator Driven System (ADS) such as the Energy Amplifier (EA) [1] has the potential to destroy all actinide elements by fission, thereby producing energy [2]. In such a scenario, long term $\left(\geq 500\right.$ years $\left.^{1}\right)$ radiotoxicity of the waste is dominated by long-lived fission fragments (LLFF) which can, in practice, only be destroyed by nuclear decay following neutron capture.

In medicine, radioactive elements are increasingly used for diagnostic, therapy and pain relieving. These elements can be produced through neutron capture on stable elements in an accelerator driven activator, as an alternative to nuclear reactor production [3], using the inverse process invoked for the destruction of LLFF.

In both cases, it is important to optimise the efficiency of the neutron capture process. The specific neutron capture rate, $\mathrm{R}_{\text {capt., }}$ can be enhanced by maximizing each of the relevant factors in $R_{\text {capt. }} \equiv \int \Phi(E, t) \sigma(E) d E$, i.e.:

(a) the neutron flux $[\Phi(E, t)]$ (Figure 1) by the choice of a dense neutron "storage" medium (lead) with high atomic mass, high neutron elastic cross section (mean free path: $\lambda_{\mathrm{el}} \sim 3 \mathrm{~cm}$ ) and high neutron transparency (the doubly magic nature of the $208 \mathrm{~Pb}$ nucleus makes natural lead one of the most transparent element below $1 \mathrm{keV})$;

(b) the capture cross section $[\sigma(E)]$ (Figure 1) of the element to be transmuted by an efficient use of resonances because of the very small lethargic steps of neutrons slowing down in lead.

The purpose of the PS211 experiment at CERN, known as TARC (Transmutation by Adiabatic Resonance Crossing), was to test directly the concept of ARC to enhance significantly the neutron capture efficiency for LLFF, an idea put forward by one of us [3]. Indeed, neutrons have a peculiar behaviour in lead:

(a) a small average lethargy $\xi$ due to the high atomic mass of lead:

$$
\xi \equiv 1+\frac{\alpha}{1-\alpha} \ln (\alpha) \approx 9.6 \times 10^{-3} \text {, where } \alpha \equiv \frac{\left(\mathrm{m}_{\mathrm{Pb}}-\mathrm{m}_{\mathrm{n}}\right)^{2}}{\left(\mathrm{~m}_{\mathrm{Pb}}+\mathrm{m}_{\mathrm{n}}\right)^{2}} ;
$$

(b) a high and nearly energy independent elastic scattering cross section;

(c) a long "storage" time because, below the capture resonances $\left(E_{n} \leq 1 \mathrm{keV}\right)$ and down to epithermal energies, the elastic scattering process is nearly isotropic and the transparency to neutrons is very high (it takes $3 \mathrm{~ms}, 1800$ scatterings and a path in lead of 60 metres to thermalize a $1 \mathrm{MeV}$ neutron).

As a result, neutrons produced by spallation at relatively high energy $\left(E_{n} \approx\right.$ few $\mathrm{MeV})$, after having been quickly moderated by $(n, x n),(n, n ')$ reactions down to energies of a few hundred $\mathrm{keV}$, will slow down quasi adiabatically with small isolethargic steps and reach the capture resonance energy of an element to be transmuted where they will have a high probability to be captured. The resonance width is usually larger than the average lethargic step. This is the case of ${ }^{99} \mathrm{Tc}$ which has a strong neutron capture resonance at $5.6 \mathrm{eV}$ (4000 barns) (Figure 1) covering four average lethargy steps. Its resonance integral is 310 barns while the cross section

\footnotetext{
1500 years is the time it takes for the radiotoxicity of the waste to reach the level of the radiotoxicity of coal ashes corresponding to the production of the same amount of energy.
} 
at thermal/epithermal neutron energies $\left(E_{n} \leq 1 \mathrm{eV}\right)$ is only about 20 barns. Neutron capture on ${ }^{99} \mathrm{Tc}\left(\mathrm{t}_{1 / 2}=2.111 \times 10^{5} \mathrm{yr}\right)$ produces ${ }^{100} \mathrm{Tc}\left(\mathrm{t}_{1 / 2}=15.8 \mathrm{~s}\right)$ which then decays to ${ }^{100} \mathrm{Ru}$, a stable element. Thus, the radiotoxicity can be eliminated in a single neutron capture and, since ${ }^{100} \mathrm{Ru}$ has a small neutron capture cross section and both ${ }^{101} \mathrm{Ru}$ and ${ }^{102} \mathrm{Ru}$ are stable, essentially no new radioactive elements are produced. ARC should be most efficient for elements with strong capture resonances, such as ${ }^{99} \mathrm{Tc}$ and ${ }^{129} \mathrm{I}$ (which represent 95\% of the total LLFF radiotoxicity inventory).

The fact that salts of these two elements are soluble in water and could contaminate the biosphere has already triggered many studies to try to destroy them by transmutation in nuclear reactors [4], [5], [6]. In order to test the new approach proposed in [3], we have performed (a) neutron fluence measurements with several complementary techniques, providing redundancy, using detectors built by the TARC Collaboration and operated over a broad neutron energy range, from thermal up to a few $\mathrm{MeV}$ and (b) neutron capture rate measurements on ${ }^{99} \mathrm{Tc}$ [both integral and differential measurements], on ${ }^{129} \mathrm{I}$ and ${ }^{127} \mathrm{I}$ [integral measurements]. For ${ }^{99} \mathrm{Tc}$, in addition, a high statistics measurement of the ${ }^{99} \mathrm{Tc}$ apparent neutron capture cross section was obtained up to $\sim 1 \mathrm{keV}$, below which $85 \%$ of all captures occur in a typical TARC neutron spectrum. Many other capture or fission measurements relevant to the design parameters of the EA or to various other applications were performed and will be reported in a more detailed publication [7].

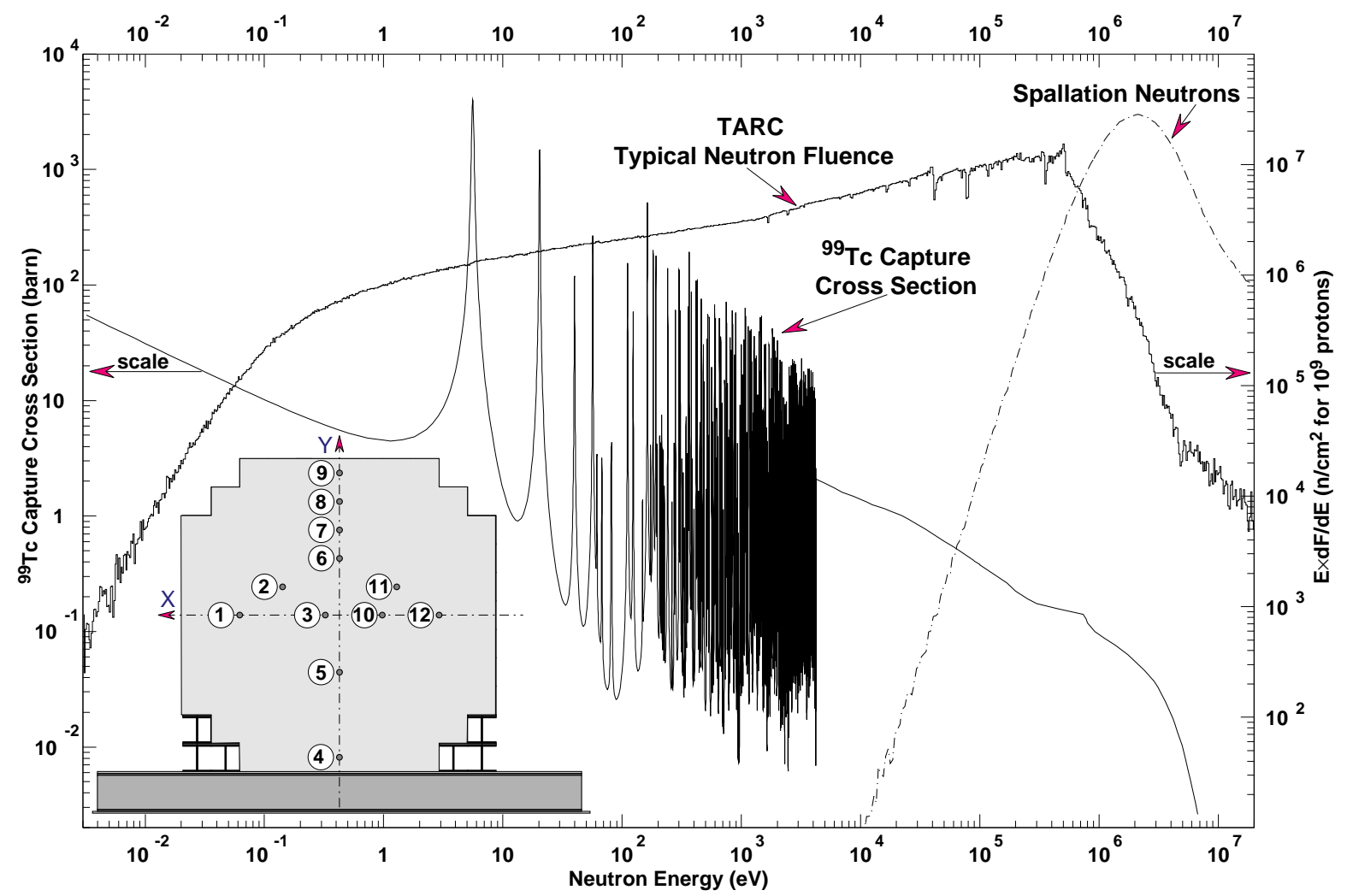

Figure 1: ${ }^{99}$ Tc neutron capture cross section (JENDL-3.2 data base [8]), as a function of neutron energy (left-hand side scale); Typical neutron fluence energy distribution in TARC, as a function of neutron energy in isolethargic bins, for $3.57 \mathrm{GeV} / \mathrm{c}$ protons (right-hand side scale); Energy distribution of spallation neutrons as they were born in lead in arbitrary units. About $14 \%$ of these neutrons are above $20 \mathrm{MeV}$. Instrumentation hole positions are shown in a sketch of the detector seen along the beam line ( $\mathrm{Z}$ axis). 


\section{The experimental set-up}

\subsection{Proton beams}

Protons from the CERN PS were provided in two different modes:

(a) fast extraction mode (20 ns long pulses; momentum of 2.5 or $3.57 \mathrm{GeV} / \mathrm{c}$; intensity ranging from $10^{7}$ to $10^{10}$ protons per pulse with a repetition time of $14.4 \mathrm{~s}$ ) used either for measurements of neutron energy from timing (the arrival time of the beam provides the origin of time), or for sample irradiation requiring high intensity. The intensity of each PS pulse was measured with beam transformers ${ }^{2}$ with an absolute precision of $\leq 5 \%$, achieved with aluminium foil and induction charge calibrations [7].

(b) slow extraction mode magnetically selecting secondary particles produced on a target made of aluminium and tungsten (spill length of $350 \mathrm{~ms}$; momentum of $2.50 \mathrm{GeV} / \mathrm{c}$ ). Individual protons were separated from other beam particles by time of flight measured with a scintillator hodoscope ( $5 \sigma$ separation of protons from other particles). This mode was used for neutron fluence measurements with ${ }^{3} \mathrm{He}$ ionization counters which required very low beam intensity (1000 protons per spill) not achievable in fast extraction mode.

\subsection{The lead volume}

Pure lead (99.99\%) [7] was chosen to ensure that impurities have a negligible effect on the neutron flux. The highest contaminant is bismuth $(19 \pm 2.8 \mathrm{ppm})$, however, with no significant effect due to its properties very close to those of lead. Among the other measurable impurities, silver plays a potentially important role because it has a very strong capture resonance at $5.2 \mathrm{eV}\left(\sigma=10^{4}\right.$ barns $)$, but fortunately below the main resonances of ${ }^{99} \mathrm{Tc}(5.6 \mathrm{eV})$ and of ${ }^{129} \mathrm{I}(70 \mathrm{eV})$. Consequently, neutrons slowing down will first go through ${ }^{99} \mathrm{Tc}$ and ${ }^{129} \mathrm{I}$ resonance energies before reaching the main resonances of silver. Nevertheless, because of the significant overlap between ${ }^{99} \mathrm{Tc}$ and silver resonances, an effort was made to have a low silver content: $3.8 \pm 0.6 \mathrm{ppm}$ (still $1.4 \%$ of all captures are expected on silver). The other detected impurities are $\mathrm{Cu}(0.1 \pm 0.2 \mathrm{ppm}), \mathrm{Cd}(0.09 \pm 0.05 \mathrm{ppm}), \mathrm{Te}(0.21 \pm$ $0.09 \mathrm{ppm}), \mathrm{Sb}(0.14 \pm 0.14 \mathrm{ppm})$ and $\mathrm{Tl}(4.6 \pm 1.4 \mathrm{ppm})$.

The 334 ton lead assembly (Figure 1) with approximate symmetry about the beam axis, was the result of an optimization between sufficient neutron containment (about $70 \%$ of all neutrons remain inside the volume), acceptable background conditions [within $1 \mathrm{~m}$ radius from the beam axis, negligible neutron background from the environment $(\leq 2 \%)]$, and affordable cost.

The beam is introduced through a $77.2 \mathrm{~mm}$ diameter blind hole, $1.2 \mathrm{~m}$ long, in such a way that the neutron shower is approximately centred in the middle of the $3 \mathrm{~m}$ long lead volume. Twelve $64 \mathrm{~mm}$ diameter instrumentation holes, parallel to the beam axis and spanning distances from 15 to $150 \mathrm{~cm}$ from the beam axis, allow the introduction of various detectors or samples for fluence or transmutation rate measurements. A more detailed description of the set-up can be found in [7].

\footnotetext{
2Beam Charge Monitor model ICT-178-070-10:1 kindly made available by Bergoz Instrumentation, Crozet, France.
} 


\section{Neutron fluence measurements}

Through the elastic scattering process in lead, a strong correlation develops between the time $t$ at which a neutron is observed and its kinetic energy $\mathrm{E}$ :

$$
E=\frac{K}{\left(t+t_{0}\right)^{2}} \text { where } K \text { is a constant }
$$

This correlation, already noted by Feinberg in 1944, has been used to measure neutron energies in so-called slowing down spectrometers [9] and is easily explained by Fermi's theory of continuous slowing down. In TARC, two of the neutron fluence detectors $\left({ }^{6} \mathrm{Li} /{ }^{233} \mathrm{U}\right.$ detectors \& ${ }^{3} \mathrm{He}$ scintillation counters) use this technique to obtain neutron energies from measurements of interaction times. The parameters $\mathrm{K}$ and $t_{0}$ of relation (2) were measured [7] with the help of a $\mathrm{CeF}_{3}$ crystal mounted in front of a photomultiplier equipped with a quartz window: this detector records the time distribution of the fast ( $5 \mathrm{~ns}$ and $30 \mathrm{~ns}$ decay times) $\mathrm{CeF}_{3}$ scintillation UV light produced by prompt $\gamma$ 's associated with radiative neutron captures on thin samples of elements with known capture resonance energies. Using eight well isolated resonances (from ${ }^{181} \mathrm{Ta},{ }^{197} \mathrm{Au},{ }^{109} \mathrm{Ag}$, ${ }^{99} \mathrm{Tc}$, ${ }^{115} \mathrm{In}$ and ${ }^{55} \mathrm{Mn}$ ) we find $\mathrm{K}=(172 \pm 2)$ $\mathrm{keV} \times \mu \mathrm{s}^{2}$ in good agreement with the simulation value $\mathrm{K}=(173 \pm 3) \mathrm{keV} \times \mu \mathrm{s}^{2}$. The parameter $\mathrm{t}_{0}=0.37 \pm 0.20 \mu \mathrm{s}$ is weakly constrained by $\mathrm{CeF}_{3}$ measurements available only over the restricted energy range $E_{\mathrm{n}} \leq 1 \mathrm{keV}$ (limited below $10 \mu$ s by the strong signal induced by the cascade of fast particles from the beam, all arriving at the same time). The systematic uncertainty on the energy due to the uncertainty in $\mathrm{K}$ and $\mathrm{t}_{0}$ is $3.5 \%$ at $1 \mathrm{keV}$ and $1.2 \%$ at thermal energy. The observed resonance widths are consistent with the expected resolution of a slowing down spectrometer given by:

$$
\frac{\sigma_{\mathrm{E}}}{\mathrm{E}} \approx 11.3 \sqrt{1+\frac{2}{\mathrm{E}(\mathrm{eV})}}(\%) \quad\left(\text { valid for } \mathrm{E}_{\mathrm{n}} \leq 1 \mathrm{keV}\right) \quad[10]
$$

We had to use several techniques in TARC to measure neutron fluences ${ }^{3}$ over the desired energy range from thermal to $\mathrm{MeV}$ neutrons.

\section{$3.1^{6} \mathrm{Li} / 233 \mathrm{U}$ detectors}

Two $10 \times 10 \times 0.3 \mathrm{~mm}^{3}$ Si detectors [7] enclosed in a common $10 \times 15 \times 20 \mathrm{~mm}^{3}$ lead box are facing respectively a ${ }^{6} \mathrm{Li}\left(100 \mu \mathrm{g} / \mathrm{cm}^{2}{ }^{6} \mathrm{LiF}\right.$ converter layer) and a $233 \mathrm{U}$ $\left(200 \mu \mathrm{g} / \mathrm{cm}^{2}\right.$ converter layer) target separated by a $0.1 \mathrm{~mm}$ thick sheet of lead. Each $\mathrm{Si}$ junction detects the ionizing products of the neutron induced reaction $n+{ }^{6} \mathrm{Li} \rightarrow \alpha$ $+\mathrm{T}+4.78 \mathrm{MeV}$ or fission fragments (FF) from 233U fissions. Diaphragms in front of them are used to remove the Si detector edge effects and particles with too large an incident angle. The $\alpha$-decay of $233 \mathrm{U}\left(\mathrm{t}_{1 / 2} \sim 1.59 \times 10^{5} \mathrm{yr}\right)$ provides an absolute calibration of the detector efficiency. This detector ensemble allows us to measure, at the same position and at the same time, the neutron fluence with two different nuclear reactions having different cross sections and sensitivities to the background. The error on the detector efficiency is $6 \%$. The detector thickness is small enough that it does not perturb the flux significantly. Flash ADC's were used to record time and amplitude of signals generated by $\alpha$ 's, T's and FF's all clearly distinguished from the observed backgrounds.

\footnotetext{
${ }^{3}$ Throughout this paper the neutron flux is always integrated over time to become a fluence per accelerator pulse, or normalized to a given number of incident protons.
} 
The measured number of events, $\Delta \mathrm{N}(\mathrm{t})$, recorded between times $\mathrm{t}$ and $\mathrm{t}+\Delta \mathrm{t}$ can be written as:

$$
\Delta \mathrm{N}(\mathrm{t})=\mathrm{n}(\mathrm{t}) \Delta \mathrm{t}=\varepsilon \mathrm{N}_{\text {at. }} \Delta \mathrm{t} \int \Phi(\mathrm{E}, \mathrm{t}) \sigma(\mathrm{E}) \mathrm{dE}=\varepsilon \mathrm{N}_{\text {at. }} \Delta \mathrm{F}(\mathrm{E}(\mathrm{t})) \int \mathrm{C}(\mathrm{E}, \mathrm{t}) \sigma(\mathrm{E}) \mathrm{dE}
$$

where $n(t)$ is the event rate, $\varepsilon$ is the detector efficiency; $N_{\text {at. }}$ is the number of atoms in the target; $\mathrm{C}(\mathrm{E}, \mathrm{t})$ is a normalized function describing the $\mathrm{E}$ dispersion at time $t$ obtained from the data; $\sigma(E)$ is the ${ }^{6} \mathrm{Li}(\mathrm{n}, \mathrm{t})$ or ${ }^{23} \mathrm{U}(\mathrm{n}, \mathrm{f})$ cross section; $\Phi(\mathrm{E}, \mathrm{t})$ is the neutron flux; $\Delta \mathrm{F}(\mathrm{E}(\mathrm{t}))$ is the fluence corresponding to all neutrons with energy in the interval $\mathrm{E}(\mathrm{t})$ and $\mathrm{E}(\mathrm{t}+\Delta \mathrm{t})$. The fluence, $\mathrm{F}(\mathrm{E})$, as a function of energy is obtained from equation (4) using the calculation of $\sigma_{\text {eff }} \equiv J \mathcal{L}(E, t) \sigma(E)$ a $E$ with the JENDL-3.2 cross section [8].

In total, 69 positions were measured [7], providing a detailed map of the neutron fluence evolution throughout the entire lead volume. Most of the data were collected using $3.57 \mathrm{GeV} / \mathrm{c}$ protons. However, 9 runs were taken with $2.5 \mathrm{GeV} / \mathrm{c}$ protons. Typical results are shown in Figures $\mathbf{2 a}$ and $\mathbf{2 b}$, in hole number $10,45 \mathrm{~cm}$ from the beam axis, at the position $\mathrm{Z}=+7.5 \mathrm{~cm}$ (Figure 1). The total systematic error on the fluence measurement is $14 \%$ below $100 \mathrm{eV}$ and reaches $16 \%$ at $1 \mathrm{keV}$.

\section{$3.2{ }^{3}$ He detector in the scintillation mode}

The scintillation light (on average 7000 photons per neutron capture on ${ }^{3} \mathrm{He}$ ) produced in a mixture of ${ }^{3} \mathrm{He}\left(1 \times 10^{5} \mathrm{~Pa}\right), \mathrm{Xe}\left(1.18 \times 10^{5} \mathrm{~Pa}\right)$ and ${ }^{4} \mathrm{He}\left(1.75 \times 10^{5} \mathrm{~Pa}\right)$ by the reaction $\mathrm{n}+{ }^{3} \mathrm{He} \rightarrow \mathrm{p}+\mathrm{T}(\mathrm{Q}-\mathrm{value}$ of $+764 \mathrm{keV})$, was detected by a phototube, after having been wave shifted to $340 \mathrm{~nm}$ in order to go through the quartz window separating the chamber from the photocathode [7] and to match the PM response. A Flash ADC was used to digitize signals from the phototube operating in the current integration mode. As in the case of the ${ }^{6} \mathrm{Li} / 233 \mathrm{U}$ detectors, the energy-time relation and the known cross section for the reaction ${ }^{3} \mathrm{He}(\mathrm{n}, \mathrm{p})^{3} \mathrm{H}$ were used to transform the measured time evolution of the interaction rate into a neutron fluence measurement as a function of neutron energy. The efficiency of the detector was continuously monitored by observing the photopeak produced by $5.5 \mathrm{MeV} \alpha$ 's from an ${ }^{241} \mathrm{Am}$ source mounted inside the chamber.

This detector covers a neutron energy range from $0.3 \mathrm{eV}$ up to about $50 \mathrm{keV}$, over which the systematic uncertainty on the neutron fluence measurement varies from $13.5 \%$ up to $55 \%$ at $50 \mathrm{keV}$. Ten positions were measured [7] within the lead volume, at each of the two proton beam momenta (3.57 and $2.5 \mathrm{GeV} / \mathrm{c}$ ). Typical results are shown in Figures $\mathbf{2 a}$ and $\mathbf{2 b}$.

\subsection{Fluence measurements at specific neutron energies}

Triple foil activation is a standard technique [7] to obtain the neutron fluence at one specific energy corresponding to a very strong isolated capture resonance in elements such as ${ }^{197} \mathrm{Au}(4.9 \mathrm{eV}), 186 \mathrm{~W}(18.8 \mathrm{eV})$ and ${ }^{115} \mathrm{In}(1.457 \mathrm{eV})$. Sandwiches of three foils of the same material are activated. The foil thickness is chosen (gold: 25 $\mu \mathrm{m}$; tungsten: $127 \mu \mathrm{m}$; indium: $127 \mu \mathrm{m}$ ) such that the outer foils are nearly opaque to neutrons at the resonance energy. The fluence at the energy of the resonance is obtained by comparing the activity of the centre foil to the average of the two outer foils. The precision of this method, limited by the uncertainty on neutron capture cross sections, is 15\%, except for tungsten where large discrepancies were found between different cross section data libraries (JENDL-3.2 [8] and ENDF/B-VI [11]). Data were collected at 14 positions for each of the three elements to provide an 
independent fluence measurement at energies within the range of the two previous electronic detectors. For tungsten, our measurements are clearly best described by ENDF/B-VI. Typical measurements [7] all using $3.57 \mathrm{GeV} / \mathrm{c}$ protons are shown in Figure $2 \mathbf{b}$, in excellent agreement with those from ${ }^{6} \mathrm{Li} / 233 \mathrm{U}$ and ${ }^{3} \mathrm{He}$ scintillation detectors.

\section{$3.4{ }^{3}$ He ionization detector}

These counters use the same ${ }^{3} \mathrm{He}(n, p){ }^{3} \mathrm{H}$ reaction as the ${ }^{3} \mathrm{He}$ scintillation counters, but measure the ionization charge deposited by the outgoing proton and triton in the chamber gas mixture $\left[{ }^{3} \mathrm{He}\left(4 \times 10^{5} \mathrm{~Pa}\right)\right.$, argon $\left(2 \times 10^{5} \mathrm{~Pa}\right)$ and $\mathrm{CH}_{4}$ $\left.\left(0.4 \times 10^{5} \mathrm{~Pa}\right)\right]$. They are of the Cutler-Shalev type [12] with the addition of guard electrodes and proportional wires allowing precise definition of the fiducial volume in which the $\mathrm{p}$ and $\mathrm{T}$ tracks are fully contained. They extend neutron fluence measurements from $20 \mathrm{keV}$ to $2 \mathrm{MeV}$.

The beam used in the slow extraction mode, mainly at a momentum of 2.5 $\mathrm{GeV} / \mathrm{c}$, contains $20 \%$ of protons separated from pions $(60 \%)$, muons and electrons $(20 \%)$ by time of flight measurements. Only beam particles with a four-fold coincidence in the scintillator hodoscope were accepted. The estimated accidental background is $\leq 1.6 \%$.

The counters with typical energy resolution of $6 \% \mathrm{FWHM}$ at the thermal neutron peak were tested with neutron sources $\left({ }^{252} \mathrm{Cf}\right.$ and ${ }^{241} \mathrm{Am} / \mathrm{Li}$ ) and calibrated with monoenergetic neutrons at the CEN Bordeaux Van De Graaff [7]. A detailed detector simulation was developed, the fiducial volume limit was adjusted by fitting the calibration data. The effect of neutron elastic scattering on hydrogen and ${ }^{3} \mathrm{He}$ nuclei was estimated to be acceptably small $(<20 \%)$ for the neutron energy range considered. Fluence energy spectra were obtained from the recorded anode signal amplitude histogram by deconvolution, taking into account the detector response function. The typical fluence uncertainty is 16 to $18 \%$, of which $15 \%$ comes from the calibration and $10 \%$ from the deconvolution process.

There has been in total more than 400 runs covering 15 positions in the lead volume [7]. A typical measurement is shown in Figures $\mathbf{2 a}$ and $\mathbf{2 b}$.

\subsection{Other checks of the neutron fluence}

Several additional cross-checks of the neutron fluence were performed outside the energy range covered by the electronic detectors. At low energies, the standard thermoluminescence technique was used [7]. At high energies, in the energy range dominated by the spallation process, measurements of the fission rate in ${ }^{232} \mathrm{Th}$ samples $\left(E_{n}>1.4 \mathrm{MeV}\right)$ [7] and of threshold reaction rates $\left[{ }^{12} \mathrm{C}(\mathrm{n}, 2 \mathrm{n})^{11} \mathrm{C}\left(\mathrm{E}_{\mathrm{n}}>22\right.\right.$ $\mathrm{MeV})$ and ${ }^{12} \mathrm{C}(\mathrm{n}, 3 \mathrm{n}){ }^{10} \mathrm{C}\left(\mathrm{E}_{\mathrm{n}}>34 \mathrm{MeV}\right)$ ] [7] were performed. In all cases, a good agreement was found between data and simulation. The spatial distribution of the high energy component of the neutron fluence shows a significant forwardbackward asymmetry and dies away quickly (within $50 \mathrm{~cm}$ from the centre of the cascade generated by the beam protons), which is a very useful practical consideration for the design of the EA where fast neutron damage in structural materials must be minimized. 

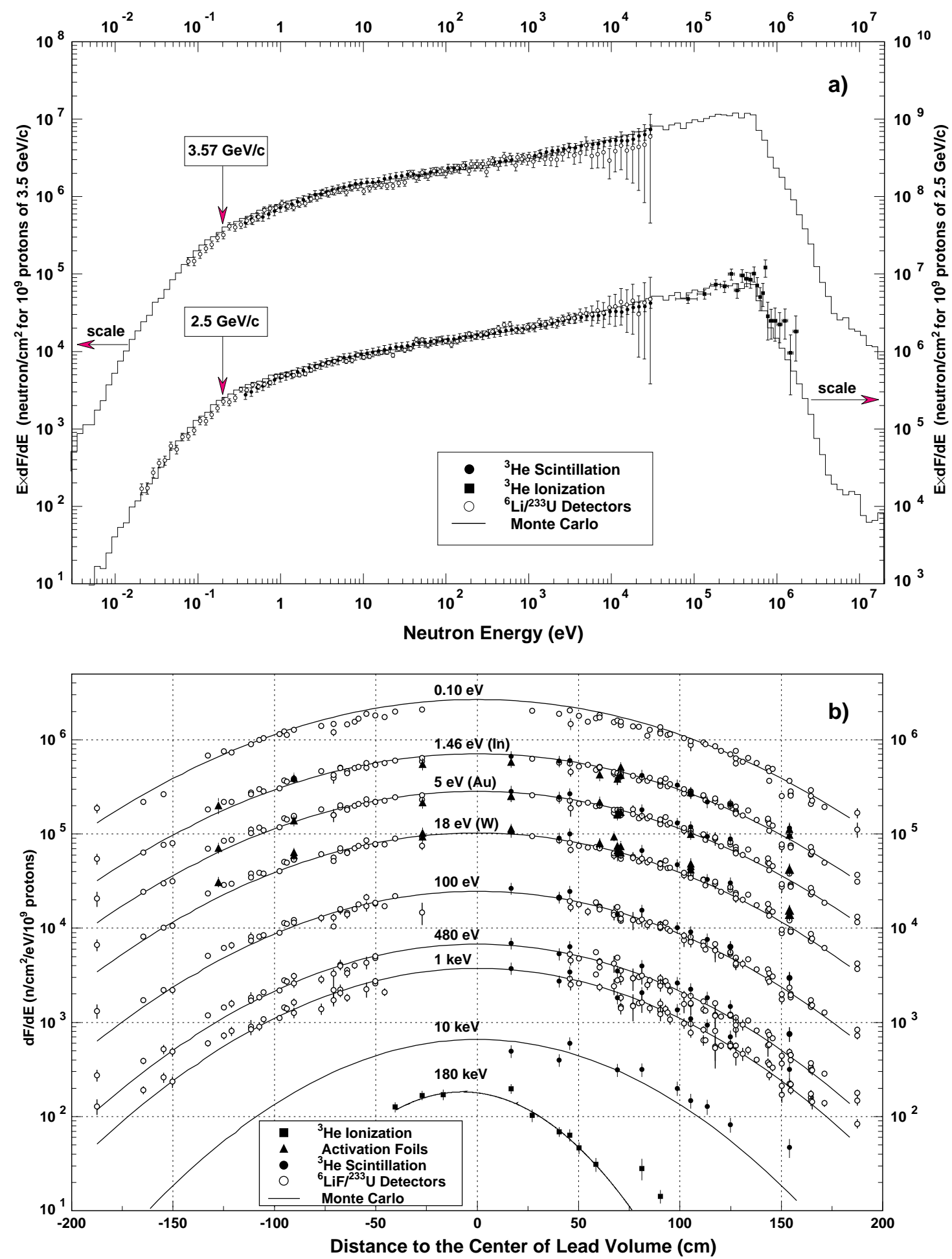

Figure 2: a) Example of TARC measurements of the neutron fluence. $\mathrm{E} \times \mathrm{dF} / \mathrm{dE}$ is shown as a function of neutron energy, from 2.5 and $3.57 \mathrm{GeV} / \mathrm{c}$ protons in hole number $10(\mathrm{Z}=7.5 \mathrm{~cm}$, at a distance of $45.6 \mathrm{~cm}$ from the centre of the lead volume). The Monte Carlo predictions are shown as histograms. The data are from ${ }^{6} \mathrm{Li} /{ }^{233} \mathrm{U}$ detectors (open circles), ${ }^{3} \mathrm{He}$ in the scintillation mode (full circles) and ${ }^{3} \mathrm{He}$ in the ionization mode (full squares). The error bars include both statistical and systematic errors added in quadrature;

b) Variation of the neutron fluence as a function of distance from the centre of the lead volume (the minus sign indicates negative $\mathrm{Z}$ positions). $\mathrm{dF} / \mathrm{dE}$ is shown for a few selected energy bins. The Monte Carlo predictions are shown as lines obtained from a Gaussian fit to Monte Carlo points. The data are shown with the same convention as in part a) with in addition, data from triple foil activations shown as full triangles. 
Furthermore, the total energy deposited by neutrons in the lead, which is related to the neutron fluence, was obtained from "thermometer" measurements [7] near the spallation region and found to agree with the Monte Carlo simulation. The temperature evolution with time of a small, thermally isolated lead sample prepared at liquid helium temperature $(4.15 \mathrm{~K})$ was measured with a resolution of $0.2 \mathrm{mK}$ as a function of neutron irradiation.
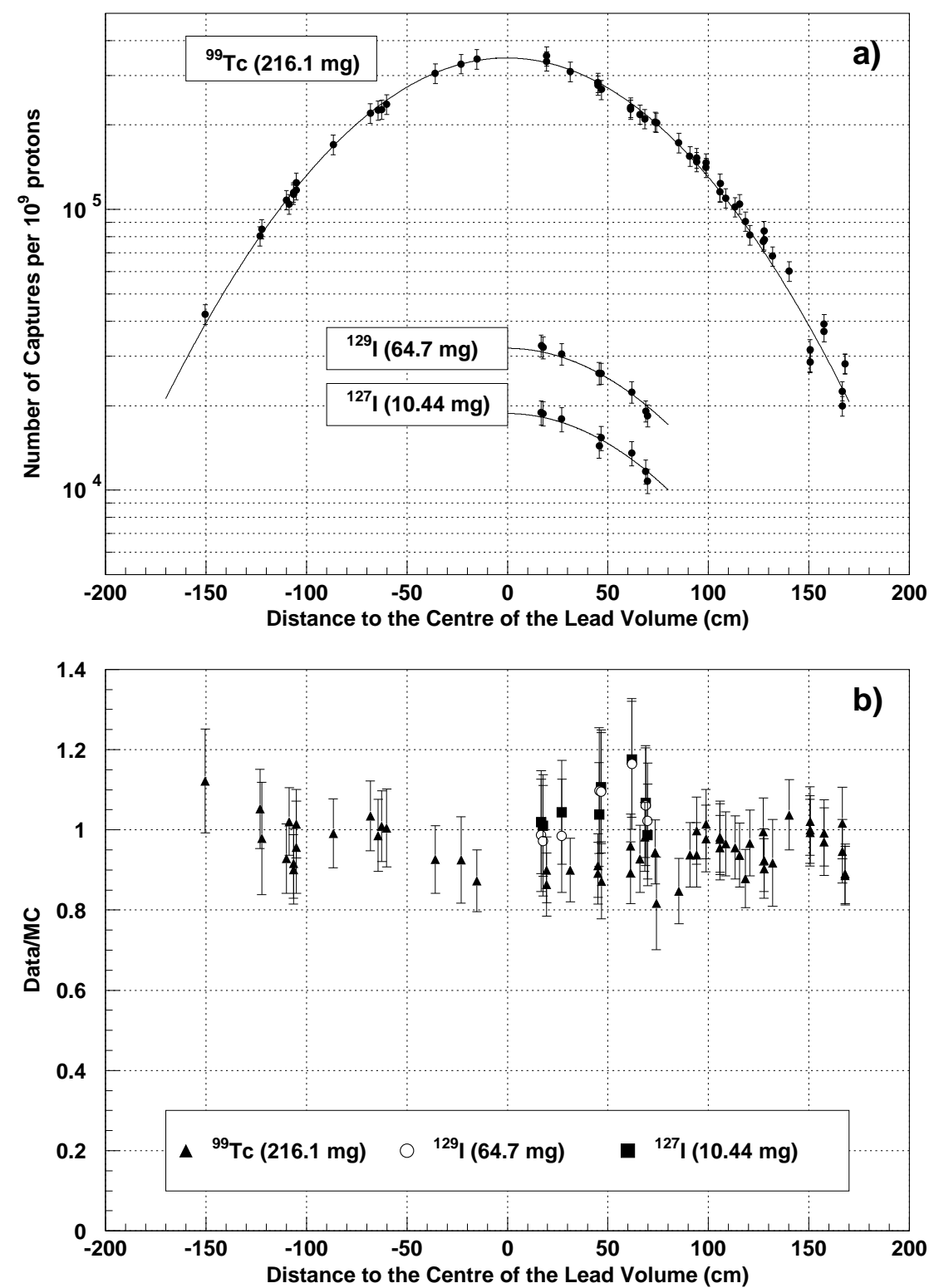

Figure 3: a) Measurement of transmutation rates for ${ }^{99} \mathrm{Tc}(216.11 \mathrm{mg}),{ }^{129} \mathrm{I}(64.7 \mathrm{mg})$ and ${ }^{127} \mathrm{I}$ (10.44 mg) samples as a function of distance from the centre of the lead volume. The lines are Gaussian fits to the data;

b) Ratio between data and prediction for the transmutation rates shown in part a).

In summary, we obtained a large coherent set of neutron fluence measurements with redundant methods especially, in the energy range below $\sim 10 \mathrm{keV}$ where we want to use the ARC method. We verified that $d F / d[\ln (E)]$ decreases very slowly with neutron energy, only by one order of magnitude in the neutron energy range from $10 \mathrm{keV}$ to $1 \mathrm{eV}$. These neutron fluence measurements constitute the first important element in our demonstration of the efficiency of the ARC effect. In the following section we describe a more direct test on LLFF. 


\section{Transmutation rates for technetium $\left({ }^{99} \mathrm{Tc}\right)$ and iodine $\left({ }^{129} \mathrm{I} \&{ }^{127} \mathrm{I}\right)$}

\subsection{Technetium: ${ }^{99} \mathrm{Tc}$}

We measured the transmutation rate of ${ }^{99} \mathrm{Tc}$ and ${ }^{129} \mathrm{I}$, placed in the accurately known neutron fluence of TARC, using $3.57 \mathrm{GeV} / \mathrm{c}$ protons. For ${ }^{99} \mathrm{Tc}$ we used a "rabbit" pneumatic system [7], allowing us to position precisely a ${ }^{99} \mathrm{Tc}$ sample contained in a small low-activation carbon fibre shuttle, at a given location within the lead volume. After $0.5 \mathrm{~s}$ exposure to neutrons produced by the beam, the shuttle is transported quickly (1.2 s) over 24 metres to a precise position between two high purity Ge counters to detect during 9 seconds the $\gamma$ s associated with the decay of the produced ${ }^{100} \mathrm{Tc}$. Just before the next PS proton pulse comes in, the shuttle is sent back to the irradiation position. The PS cycle time of $14.4 \mathrm{~s}$ is well matched to the $15.8 \mathrm{~s}$ half-life of ${ }^{100} \mathrm{Tc}$, and with the above procedure, high statistics data were collected from many PS pulses, until typically $2 \times 10^{4}$ counts were recorded in each of the two main $\gamma$ lines of interest (539 and $590 \mathrm{keV}$ ). A major effort was made to have negligible $\gamma$ background from the environment, to handle the strong $\beta$ radiation from the ${ }^{99} \mathrm{Tc}$ sample itself (optimized filter) and to build a reliable "rabbit" system, allowing us to collect very large statistics data at each measured position. Using two Ge counters, both measuring the ${ }^{99} \mathrm{Tc}$ activation between PS pulses, checking that the two main ${ }^{99} \mathrm{Tc} \gamma$ lines gave the same result, using different types of ${ }^{99} \mathrm{Tc}$ samples $\left[\mathrm{K}^{99} \mathrm{TcO}_{4}\left(0.1009 \mathrm{~g}\right.\right.$ and $0.2161 \mathrm{~g}$ of $\left.{ }^{99} \mathrm{Tc}\right)$; metallic $\left.{ }^{99} \mathrm{Tc}(14.3 \mathrm{mg})\right]$ and other known materials such as natural $\mathrm{Al}$ and $\mathrm{Ag}$ was part of the procedure to control carefully the systematics of the measurements.

In hole number 10 at $\mathrm{Z}=+7.5 \mathrm{~cm}$, with the $\mathrm{K}^{99} \mathrm{TcO}_{4}$ sample containing $0.2161 \mathrm{~g}$ of ${ }^{99} \mathrm{Tc}$, the ${ }^{99} \mathrm{Tc}$ capture rate is $(2.67 \pm 0.43) \times 10^{5}$ per $10^{9}$ protons [7]. The experimental precision in the measurement of the $\gamma$ rate from ${ }^{100} \mathrm{Tc}$ decay is $8.2 \%$, but the resulting uncertainty on the ${ }^{99} \mathrm{Tc}$ transmutation rate is $16 \%$, of which a global error of $14 \%$ comes from the uncertainty in the fraction of ${ }^{100} \mathrm{Tc}$ decays producing $\gamma$ 's (BR 7\% ) [13]. About 65\% of ${ }^{99} \mathrm{Tc}$ captures occur at the 5.8 and $22.3 \mathrm{eV}$ resonances, a fraction which varies with distance (50 and $80 \%$ respectively for near and far positions in TARC). A global map (60 positions) of the capture rate in the lead volume was obtained (Figure 3a), showing that the distribution within a radius of 1.5 metres from the centre of the lead volume has a high spherical symmetry, as in the case of the spatial distribution of the neutron fluence at low energy (Figure $\mathbf{2 b}$ ). Because of the small elastic collision length of neutrons in lead $\left(\lambda_{\mathrm{el}}=3 \mathrm{~cm}\right)$, the detailed shape of the neutron spallation cascade is rapidly forgotten during the moderation process. The observed transmutation rates are in excellent agreement with the predictions (Figure 3b).

With the $\mathrm{CeF}_{3}$ crystal used to calibrate the energy-time relation, we obtained measurements of the ${ }^{99} \mathrm{Tc}$ transmutation rate as a function of neutron energy (Figure 4) from the detection of prompt $\gamma$ 's associated to neutron captures. The sample used contained $85 \mathrm{mg}$ of ${ }^{99} \mathrm{Tc}$ in a $1.7 \mathrm{~g}$ aluminium matrix of $4 \mathrm{~cm}^{2}$ area. Background measurements were made using a pure aluminium sample of identical dimensions. These differential transmutation measurements also agree well with our simulation (with the evaluated cross section from the JENDL-3.2 data base [8]) and provide, after dividing by the neutron fluence, a high statistics measurement of the ${ }^{99} \mathrm{Tc}$ apparent capture cross section up to about $1 \mathrm{keV}$, with an overall systematic uncertainty ranging from $22 \%$ at low energy up to $26 \%$ at $1 \mathrm{keV}$. In this analysis, we assumed that the efficiency for the detection of the $\gamma$ cascade is independent of 
neutron energy, which seems reasonable since, up to a few $\mathrm{keV}$, the neutron angular momentum with respect to the target nucleus remains mostly $\mathrm{L}=0$. The $\mathrm{CeF}_{3}$ detector absolute efficiency as a function of energy was checked with a Monte Carlo simulation, but we used the "rabbit" integrated capture rate measurement to provide the absolute normalization. We did not extrapolate our cross section to zero thickness because the ${ }^{99} \mathrm{Tc}$ sample thickness is small $\left(21.2 \mathrm{mg} / \mathrm{cm}^{2}\right)$, and the corresponding correction, except at the 5.6 and $22.3 \mathrm{eV}$ resonances where it is $50 \%$ and $18 \%$ respectively, is well within the overall systematic error. The previous measurement of Chou et al. [10], presently used in cross section evaluations, is supposed to be extrapolated to zero thickness, yet figure 4 shows that those data taken with relatively thick targets are significantly lower than our non-corrected data on the first two resonances.

A study of ${ }^{99} \mathrm{Tc}$ resonances at Geel [14] using time of flight techniques has been reported recently, implying only a $+3 \%$ change with respect to JENDL-3.2, in the prediction of the number of ${ }^{99} \mathrm{Tc}$ captures at $\mathrm{Z}=+7.5 \mathrm{~cm}$ in hole 10 , when integrating over the common energy range of 0.7 to $617 \mathrm{eV}$ [15].

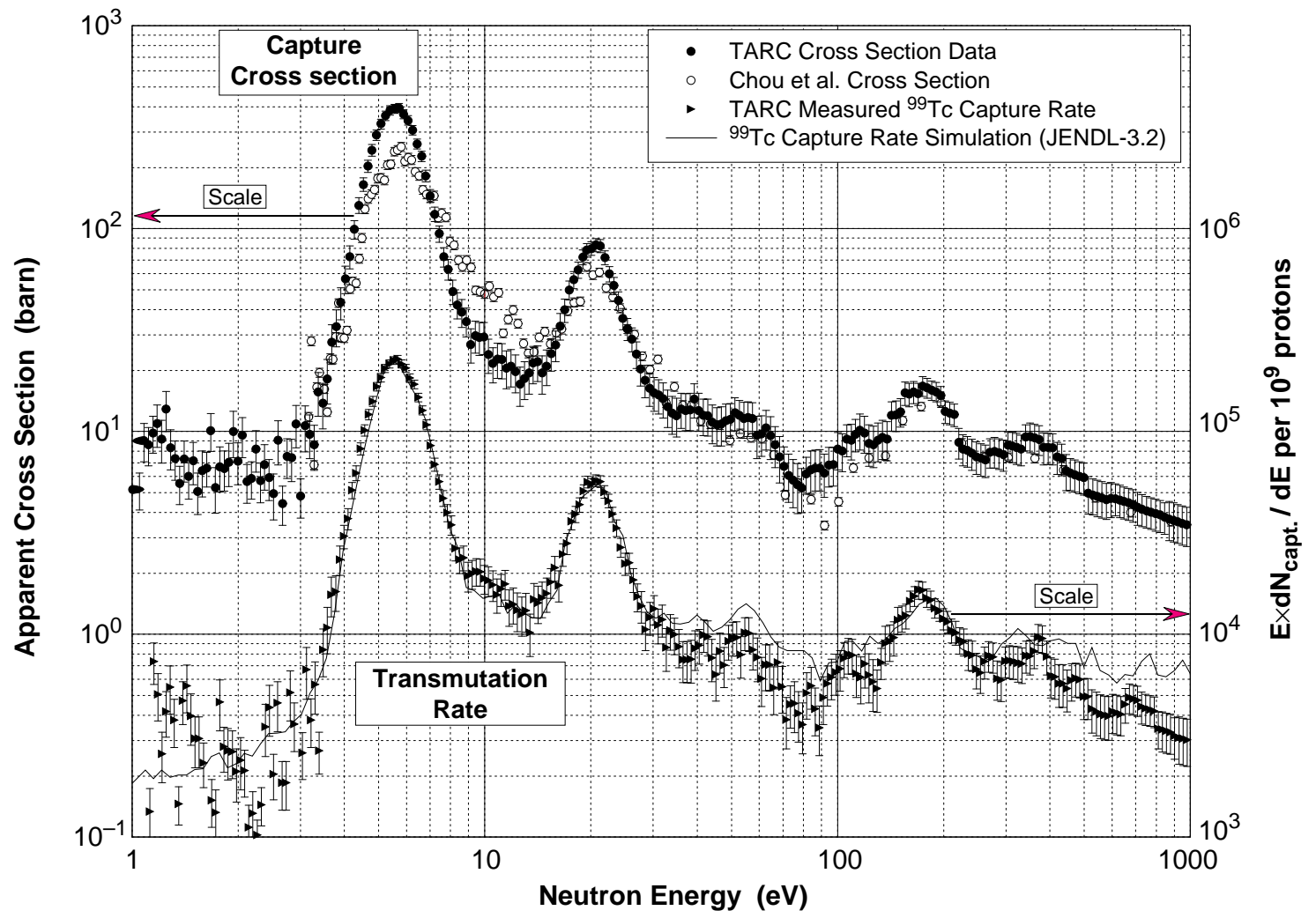

Figure 4: Measured transmutation rate (hole $10, \mathrm{Z}=+7.5 \mathrm{~cm}$ ) for a $85 \mathrm{mg}{ }^{99} \mathrm{Tc}$ sample as a function of neutron energy compared to simulation (right-hand scale). Measurement of the ${ }^{99} \mathrm{Tc}$ apparent neutron capture cross section as a function of neutron energy, and comparison with the previous measurement by Chou et al. [10] extrapolated to zero thickness (left-hand scale).

\subsection{Iodine: ${ }^{129}$ I and ${ }^{127}$ I}

The other main LLFF studied in TARC is ${ }^{129} \mathrm{I}$. A (64.7 \pm 1.0$) \mathrm{mg}$ sample of ${ }^{129} \mathrm{I}$ in the form of $\mathrm{PbI}_{2}$ was irradiated at five different positions in the TARC volume [7], using $3.57 \mathrm{GeV} / \mathrm{c}$ protons. We used the $13.9 \%$ of $127 \mathrm{I}(10.44 \pm 0.2) \mathrm{mg}$ present in the sample to measure at the same time the capture rate on natural iodine, relevant for the production of radioactive isotopes (i.e. ${ }^{128}$ I) for medical applications [3]. 
The capture process in the case of ${ }^{129} \mathrm{I}$ produces either the isomer $130 \mathrm{mI}^{\mathrm{I}}\left(\mathrm{t}_{1 / 2}=\right.$ $9 \mathrm{~m})$ or the ground state ${ }^{130 \mathrm{gI}}\left(\mathrm{t}_{1 / 2}=12.36 \mathrm{~h}\right)$ leading to ${ }^{130} \mathrm{Xe}$, a stable element. It is therefore necessary to measure both the isomer and the ground state production, and take into account transitions from isomer to ground state. We find a ratio isomer over ground state of $1.55 \pm 0.15$ in good agreement with a previous MIT measurement [16]. The total uncertainty on the ${ }^{129} \mathrm{I}$ and ${ }^{127} \mathrm{I}$ capture rates is $10 \%$. The capture rates we measured in hole number 10 at $Z=+7.5 \mathrm{~cm}$ are $26100 \pm 2600$ per $10^{9}$ protons for ${ }^{129} \mathrm{I}$ and $14900 \pm 1500$ for ${ }^{127}$ I. The transmutation rates of both ${ }^{129} \mathrm{I}$ and ${ }^{127} \mathrm{I}$ are in excellent agreement with our simulation (Figures $3 \mathbf{a}$ and $\mathbf{3 b}$ ).

In summary, the observed transmutation rates $\left({ }^{99} \mathrm{Tc}\right.$ and $\left.{ }^{129} \mathrm{I}\right)$ both agree well with the prediction and follow very closely the decrease of neutron fluence as a function of increasing distances from the centre of the lead volume (Figures $\mathbf{2 b}$ and 3a). This implies that neutron capture at low energies dominates and that the capture rate per unit of fluence varies little within most of the lead volume.

\section{Comparison of data with the Monte Carlo simulation}

A special effort was made, in the framework of the EA programme, to develop an innovative simulation [7] which has taken up the most challenging approach to the problem of neutron transport and burnup simulation by integrating transport of the neutrons generated in the spallation process and evolution of material composition under the combined effects of neutron activation and nuclear decay. We now have a unique tool which combines the full precision of the Monte Carlo method with high simulation speed and an integrated time-evolution during burnup. The spallation process itself was simulated with FLUKA-97 [17], one of the best Monte Carlo codes available for hadronic interactions at intermediate energies. In particular, the complex structure of cross sections in the resonance region requires careful treatment to describe correctly self-shielding effects. In practice, the TARC simulation was carried out in two steps: (a) the spallation process and neutron transport down to $19.6 \mathrm{MeV}$, using FLUKA; (b) further transport of neutrons all the way until they either escape the lead volume or are captured, using a new simulation code [7].

Both the energy and space distributions and the absolute magnitude of the neutron fluence throughout the entire lead volume are well described by the simulation. For instance, neutron fluence data from thermal energies up to $2 \mathrm{MeV}$, over eight orders of magnitude, are very well reproduced at both proton beam energies (Figure 2a). We find that about $70 \%$ of the spallation neutrons survive the lead capture resonances. The ratio of fluences (between $0.1 \mathrm{eV}$ and $10 \mathrm{keV}$ ) obtained with two proton momenta ( 3.57 and $2.5 \mathrm{GeV} / \mathrm{c}$ ) is found to be $1.52 \pm 0.1$ consistent with the expectation from the simulation: $1.57 \pm 0.01$ (stat.) which is essentially the ratio of kinetic energies of the beams. The global uncertainty in the Monte Carlo prediction comes mainly from the uncertainties in the spallation process (difficult to quantify exactly but globally estimated at $10 \%$ ) and in the neutron transport in lead (mainly the uncertainty on the lead cross section of 10\%) and amounts to a total of $15 \%$. Note that the impurity content is small enough, with well measured concentrations, so that the corresponding uncertainty on the neutron fluence is negligible [7].

The excellent agreement with the entire TARC data set validates in detail our simulation. It confirms in particular that the spallation process is correctly predicted 
within the errors mentioned above. Typically, we expect 100 neutrons per 3.57 $\mathrm{GeV} / \mathrm{c}$ proton. The dependence of the neutron fluence on energy and space validates the neutron transport code and tests the reliability of the lead cross sections. The correct prediction of integral and differential transmutation rates for ${ }^{99} \mathrm{Tc}$ and ${ }^{129} \mathrm{I}$ validates the efficiency of the TARC method to transmute LLFF.

\section{Practical scheme for the transmutation of LLFF}

The simulation validated by TARC was then used to predict the transmutation rate for ${ }^{99} \mathrm{Tc}$ [3], in the lead volume outside the EA core, where the neutron fluence is quasi isolethargic, precisely as in TARC. It is important to note that the goal of TARC was to collect basic data on transmutation but not to carry out transmutation in an optimized way. Furthermore, the transmutation rates obtained cannot be directly extrapolated to larger amounts of material on the industrial scale, mainly because resonance self-shielding effects are important. Such effects have been observed in TARC, where three different ${ }^{99} \mathrm{Tc}$ samples gave different transmutation rates per gram of ${ }^{99} \mathrm{Tc}$ (i.e. in hole number 10 at $\mathrm{Z}=+7.5 \mathrm{~cm}$ and for $10^{9}$ protons of 3.57 $\mathrm{GeV} / \mathrm{c}$ momentum, we have $2.34 \times 10^{6}, 1.52 \times 10^{6}$ and $1.24 \times 10^{6}$ captures / $g$ respectively for $14.3,100.9$ and $216.1 \mathrm{mg}$ of ${ }^{99} \mathrm{Tc}$ ) all well reproduced by the simulation [7].

For instance, the TARC simulation predicts that, in a $1 \mathrm{GW}_{\text {th }} \mathrm{EA}$, after a burnup of $100 \mathrm{GW}_{\mathrm{th}} \times \mathrm{d} / \mathrm{t}$ and with an initial load of $270 \mathrm{~kg}$ of ${ }^{99} \mathrm{Tc}$ uniformly diluted in lead at a mass concentration of $1.04 \times 10^{-3}$, the fraction of ${ }^{99} \mathrm{Tc}$ transmuted is $16.5 \%$ [3]. This corresponds to a transmutation rate of $1.4 \mathrm{~kg} / \mathrm{TW}_{\mathrm{th}} \times \mathrm{h}$ to be compared to the production rate of $0.85 \mathrm{~kg} / \mathrm{TW}_{\mathrm{th}} \times \mathrm{h}$ in the EA. With a mass concentration of $1.8 \times 10^{-3}$ for ${ }^{129} \mathrm{I}$ and an initial load of $430 \mathrm{~kg}$, one obtains a ${ }^{129} \mathrm{I}$ burning rate of $1 \mathrm{~kg} / \mathrm{TW}_{\mathrm{th}} \times \mathrm{h}$ to be compared to a production rate of $0.4 \mathrm{~kg} / \mathrm{TW}_{\mathrm{th}} \times \mathrm{h}$ in the EA.

The fact that this takes place outside the EA core, in a region where there is a small probability that neutrons will return to the core and be useful for fissions, makes it possible to envisage LLFF transmutation in a parasitic mode, thus minimizing the cost of the process. We find it a very attractive idea to use the excess of neutrons outside the EA core to reduce the radiotoxicity produced in the overall fuel cycle instead of letting them eventually contribute to the activation of the surroundings.

\section{Conclusion}

We have obtained a large coherent set of neutron fluence data over 8 orders of magnitude in neutron energies (from thermal to $2 \mathrm{MeV}$ ). In the energy region below $10 \mathrm{keV}$ this was done with three different detector techniques, each with an absolute precision of the order of $15 \%$. The spatial distribution of the neutron fluence within the lead volume has been mapped in detail, over distances of up to 1.9 metres from the centre.

At low energies, below $\sim 10 \mathrm{keV}$ and up to a radius of 1.5 metres, the neutron fluence, in good approximation, has spherical symmetry and behaves approximately as for a point-like source, even though neutron production through the spallation process is neither point-like nor isotropic. This is of course precisely what is expected from the small elastic collision length in lead and also from the fact that the lead volume is sufficiently large that edge effects do not constrain the shape of the neutron cloud. The $\mathrm{dF} / \mathrm{d}[\ln (\mathrm{E})]$ distribution (Figure 2a) decreases very slowly, only one order of magnitude when the neutron energy decreases by four orders of 
magnitude (from $10 \mathrm{keV}$ to $1 \mathrm{eV}$ ). This is the first important element in the demonstration of the efficiency of ARC.

The many measurements performed with ${ }^{99} \mathrm{Tc},{ }^{129} \mathrm{I}$ and $127 \mathrm{I}$ throughout the lead volume provide self-consistency checks (reproducibility of measurements, control of systematics) and confirm the validity of ${ }^{99} \mathrm{Tc}$ and ${ }^{129} \mathrm{I}$ cross section data. From the excellent agreement with the simulation, we conclude that the ARC effect is well understood.

The comprehensive ensemble of measurements reported here constitutes a very precise validation of the innovative simulation developed by the TARC Collaboration, with which practical schemes for the transmutation of LLFF were studied. We limited the present discussion to a conceptual study. Whether or not in practice ${ }^{99} \mathrm{Tc}$ or ${ }^{129} \mathrm{I}$ is diluted in lead or introduced in another form belongs to an engineering study. However, on the basis of the TARC results, it appears possible to destroy, outside the EA core and in a parasitic mode, very large amounts of ${ }^{99} \mathrm{Tc}$ or ${ }^{129} \mathrm{I}$ at a rate exceeding the production rate, thereby making it practical to reduce correspondingly the existing stockpile of LLFF. The TARC experiment also demonstrates the possibility of producing radioactive elements in particular for medical applications (as shown directly with the 127I activation measurement), with an accelerator driven activator, which constitutes an attractive alternative to the production with nuclear reactors.

\section{Acknowledgements}

We would like to thank the DGXII of the European Union for their strong financial support (contract F141-CT96-009) and M. Hugon for his excellent coordination of the P\&T cluster. The enthusiastic support of the CERN PS Division in setting up the beam, the precious help of the TIS Division for the handling of radioactive samples and the professional contribution of the ECP Division to the preparation of our data acquisition system were greatly appreciated. We would like to acknowledge the support of the various other funding institutions without which this experiment would not have been possible: Centre National de Recherche Scientifique and IN2P3 in France, Empresa Nacional de Residuos Radiactivos S.A., Comisión Interministerial de Ciencia y Tecnología and the Agrupación Eléctrica para el Desarrollo de la Tecnología Nuclear in Spain. We would like to thank M. Schneegans from LAPP for providing the $\mathrm{CeF}_{3}$ scintillator and A. Leprêtre from CEA Saclay for providing some of the ${ }^{99} \mathrm{Tc}$ targets. We would like to acknowledge also the precious help we received from A. Ferrari and P. Sala who made the latest version of FLUKA available to us, and helped us with some aspects of the simulation. Finally, we would like to thank Z. X. Zhao from CIAE for his contribution during his stay at CERN.

\section{References}

[1] C. Rubbia et al., "Conceptual Design of a Fast Neutron Operated High Power Energy Amplifier", CERN/AT/95-44 (ET), Sept. 29, 1995; C. Rubbia, "A High Gain Energy Amplifier Operated with fast Neutrons", AIP Conference Proc. 346, Int. Conf. on ADT Technologies and Applications, Las Vegas, 1994.

[2] C. Rubbia, S. Buono, Y. Kadi and J.A. Rubio, "Fast Neutron Incineration in the Energy Amplifier as Alternative to Geological Storage: the Case of Spain", CERN/LHC/97-01 (EET). 
[3] C. Rubbia, "Resonance Enhanced Neutron Captures for Element Activation and Waste Transmutation", CERN/LHC/97-04 (EET).

[4] D. W. Wootan et al., Trans. Amm. Nucl. Soc., 64, 125 (1991).

[5] R. J. M. Konings et al., J. of Nucl. Mat. 254 (1998) 122-128.

[6] R. J. M. Konings, J. of Nucl. Mat. 244 (1997) 16-21.

[7] The TARC Collaboration, "Neutron Driven Nuclear Transmutation by Adiabatic Resonance Crossing", to be submitted to NIM and references therein.

[8] JENDL-3.2 Data Base, OECD/NEA Data Bank, Issy-Les-Moulineaux, Paris, France, 1994.

[9] A. A. Bergman, A. I. Isakov, I. D. Murin, F. L. Shapiro, I. V. Stranikh, M. V. Kazarnovsky, "Lead Slowing-Down Neutron Spectrometry", Proc. 1st Int. Conf. on Peaceful Uses At. Energy, Vol. 4, 1955, p.135.

[10] J-C. Chou and H. Werle, Journal of Nucl. Energy, Vol. 27, pp. 811 to 823, Pergamon Press 1973 and data from www.nndc.bnl.gov/nndc/exfor/.

[11] ENDF/B-VI Data Base, OECD/NEA Data Bank, Issy-Les-Moulineaux, Paris, France, 1994.

[12] S. Shalev and J.M. Cutler, Nucl. Sci. Eng. 51, (1973) p. 52.

[13] G. Berzins et al., Phys. Rev. 187 (1969) p. 1618.

[14] F. Gunsing et al., Proc. Nuclear Data for Science and Technology, SIF Bologna (1997) p 1293 and private communication.

[15] The ${ }^{99}$ Tc capture cross section based on Geel data was evaluated by Z. X. Zhao, using the SAMMY-K1 code ORNL/TM-9179/R3, 1996.

[16] P. K. Hopke et al., Phys. Rev. C Vol 8 (1973).

[17] A. Ferrari and P. R. Sala, Proc. of Int. Conf. on Nuclear Data for Science and Technology, NDST-97, Trieste, Italy, 1997, ed. Italian Phys. Soc. Vol. 59, Part I, p. 247. 